\title{
OVERVIEW OF THYROIDOLOGY
}

\author{
Mohammed Moinuddin, M.D., F.A.C.P.
}

Associate Chief, Nuclear Medicine Dept.

Baptist Memorial Hospital, Memphis, Tenn.

Associate Clinical Professor in Radiology (Nuclear Medicine)

University of Tennessee, Memphis, Tenn.

Clinical Asst. Professor of Internal Medicine

Dept. of Internal Medicine

University of Tennessee, Memphis, Tenn.

DOI: http://dx.doi.org/10.5915/13-4-11970

\section{INTRODUCTION:}

Multiplicity of Thyroid tests has added a lot of confusion to the clinicians who do not deal with thyroid problems everyday. As new tests are being added to the armamentarium of diagnostic work-up, it has become difficult for the non-thyroidologists to keep up with the modern advances in Thyroidology. The object of this overview is to provide the physicians with a sufficient working knowledge about the diagnosis of patients with thyroid disorders and their management. Although it is true in every other area in medicine, it is particularly more so in thyroidology, that a thorough understanding of physiology is extremely important to appreciate the relevance of different thyroid function tests in different thyroid diseases. Therefore, this overview will start with a brief review of thyroid physiology.

\section{Thyroid Physiology}

The important thyroid hormones are Thyroxine ( $\left.\mathrm{T}_{4}\right)$ and Triiodothyronine $\left(\mathrm{T}_{3}\right) . \mathrm{T}_{3}$ is the metabolically active hormone whereas $\mathrm{T} 4$ is usually referred to as prohormone because its metabolic activity is generated only after its conversion to $T_{3}$. There is some evidence, however, that $\mathrm{T}_{4}$ by itself has weak action in large amounts. Thyroid gland is under the control of Thyroid Stimulating Hormone (TSH) secreted by thyrotrope cells of anterior pituitary. Hypothalamus secretes a tripeptide, Thyrotropin Releasing Hormone (TRH) which stimulates TSH which in turn stimulates the adrenylcyclase system in Thyroid to produce $\mathrm{T}_{3}$ and $\mathrm{T}_{4} . \mathrm{T}_{3}$ then suppresses TSH thus maintaining homeostasis. What stimulates or inhibits TRH is not clearly known.

In the United States there is daily consumption of $300-700$ ug of lodine in the diet. This is absorbed in the G.1. tract and enters the follicular cell of thyroid by a process of active transport. After this trapping. Iodine immediately undergoes oxidation by Peroxidase Enzyme system to organic iodine. It then combines with tyrosine molecule of a large Glycoprotein,
Thyroglobulin which is present in the colloid portion of thyroid follicle. The resultant products are monoiodotyrosine (M.I.T.) and Diiodotyrosine (D.I.T.) Coupling between these compounds follows, resulting in $\mathrm{T}_{3}$ and $\mathrm{T}_{4}$.

After the formation of $T_{3}$ and $T_{4}$, these are stored in the thyroid gland and released from time to time under the effect of TSH when the need arises. When these thyroid hormones enter the blood stream, 99.95\% are bound to proteins (TBG, Pre-albumin and Albumin) and only $0.05 \%$ hormones are in free form that diffuse across the cell membrane, In certain organs, notably the liver and brain, $\mathrm{T}_{4}$ undergoes deiodination to $\mathrm{T}_{3}$ and Reverse $\mathrm{T}_{3}\left(\mathrm{~T}_{3}\right)$. The former is an active hormone while the latter is biologically inert. About 75 to $80 \% \mathrm{~T}_{3}$ in the blood is the result of monodeiodination of $\mathrm{T}_{4}$ whereas only 20 to $25 \%$ of blood $\mathrm{T}_{3}$ is directly coming from thyroid. At a cellular level $\mathrm{T}_{3}$ attaches to the receptor sites on the nucleus and after exerting the metabolic actions is degraded to acetic acid and pyruvic acid which are subsequently excreted in the urine. This was a brief outline of Thyroid Physiology.

\section{Thyroid Tests}

Before we go on to the discussion of thyroid diseases and selection of appropriate tests for their diagnosis, it is important to discuss the various tests utilized in clinical practice. Some of these tests are as follows:

1. $\mathrm{T}_{4}$ - This in-vitro test measures total amount of this hormone. Free $\mathrm{T}_{4}$ is now available in some of the laboratories and is generally felt to be more sensitive than total $\mathrm{T}_{4}$. However, the latter is sufficiently good test in a large majority of patients with thyroid dysfunctions. There are several drugs and non-thyroidal conditions which can alter serum $\mathrm{T}_{4}$. The most common conditions that elevate serum $\mathrm{T}_{4}$ are high $\mathrm{TBG}$-pregnancy and estrogen therapy (Birth Control pills, etc.). Dilantin, Heparin, testosterone, steroids, etc. decrease serum $T_{4}$. Since $T_{4}$ is directly proportional to TBG, the higher the TBG, the higher the 
$\mathrm{T}_{4}$ and vice versa. Therefore, this test should be combined with a test that measures the saturation of protein binding sites by thyroid hormones. This is the $\mathrm{RT}_{3} \mathrm{U}$ test.

2. $\mathrm{RT}_{3} \mathrm{U}$ measures the saturation of binding sites in proteins by thyroid hormones. It is inversely proportional to TBG. By itself, this test is extremely non-specific and should not be used. It should be combined with $\mathrm{T}_{4}$. Any non-thyroidal disease that falsely elevates the $T_{4}$ will decrease $\mathrm{RT}_{3} \mathrm{U}$ and vice versa. Therefore, the mathematical product of $\mathrm{RT}_{3} \mathrm{U}$ and $\mathrm{T}_{4}$ will be norma. Hence $\mathrm{RT}_{3} \mathrm{U}$ and $\mathrm{T}_{4}$ should be used in combination as the initial screening tests. These tests are good for diagnosing hyper-or hypothyroidism in more than $90 \%$ of cases. Free Thyroxine Index (FTI) and Effective Thyroxine Ratio (ETR) are the tests that give essentially the same information as $\mathrm{T}_{7}\left(\mathrm{RT}_{3} \mathrm{UX} \mathrm{T}_{4}\right)$ and match the free $\mathrm{T}_{4}$ closely except in a very small number of exceptions.

3. TSH. This is an important test in the early diagnosis of Primary Hypothyroidism. Because of decrease in $T_{3}$ and $T_{4}$ in Hypothyroidsim, the suppressive effect is removed and therefore Serum TSH rises. This test also differentiates known cases of Hypothyroidism between Primary and Secondary or Tertiary.

4. $\mathrm{T}_{3}$ R.1.A. This test measures total amount of triiodothyronine and should not be confused with $\mathrm{RT}_{3} \mathrm{U}$ test. $\mathrm{T}_{3} \mathrm{RIA}$ is elevated in almost all cases of Hyperthyroidism and therefore it is an extremely important test. It is the test for the diagnosis of $\mathrm{T}_{3}$ - Toxicosis in which all other tests are normal. It is not a good test for diagnosis of Hypothyroidism mainly because of two reasons:

(A) It is a last test to become abnormal ( $\mathrm{T}_{4}$ and TSH are better tests) and

(B) There are many nonthyroidal diseases that decrease $\mathrm{T}_{3}$ such as heart, lung, liver, kidney diseases, starvation, diabetes, etc.

5. Radioactive iodine uptake test. A small amount of radio-labelled iodine is given by mouth and uptake is measured in thyroid gland usually after 24 hours. A high uptake is seen in Hyperthyroidism and low in Hypothyroidism. There are again some other conditions that can cause falsely low or high uptake. lodine consumption such as in contrast radiographic tests, Thyroiditis, administration of thyroid hormones in Euthyroid individuals give low uptakes. In majority of patients, in-vitro test are sufficient to give the diagnosis, but in borderline results this test may be used to confirm the diagnosis.

6. Thyroid Scan. Morphology, size nodularity and location of thyroid scan can be studied with this test.
In addition to these commonly used tests, there is a host of other thyroid tests which are used very infrequently such as TRH-TSH test, anti thyroglobulin antibodies, TSI, Suppression and Stimulation tests, Thyroglobulin, ultrasound of neck, $\mathrm{KCLO}_{4}$ discharge test, reverse $\mathrm{T}_{3}$ etc. etc. A discussion of all these tests is beyond the scope of this overview but may be referred to in the discussion on diseases of thyroid.

In the remainder part of this presentation, various diseases of thyroid will be described with emphasis on pertinence of tests and management.

\section{Hyperthyroidism}

This condition is characterized by hyperplasia of thyroid gland with enlargement associated with excessive production of $T_{3}$ and $T_{4}$. The bottomline is the increase in basal metabolism which is reflected in several organs with corresponding symptoms. These are weight loss despite good appetite, palpitation, tachycardia, nervousness, sweating, heat intolerance, tremors, diarrhea, alopecia, eye signs, moist wet skin, restlessness etc. etc. The diagnosis is easy in most of the young patients but older patients may not have all the above symptoms and require a high index of suspicion for the diagnosis. Apathetic Hyperthyroidism is common in patients more than 50 years of age. Diagnosis is confirmed by routine screening in vitro tests $-\mathrm{RT}_{3} \mathrm{U}$ and $\mathrm{T}_{4}$. About $5 \%$ patients with Hyperthyroidism have only $\mathrm{T}_{3}$-Toxicosis, therefore $\mathrm{T}_{3}$ in serum should be determined if $\mathrm{T}_{4}$ is normal. Twenty-four hour radioactive iodine uptake ( $\mathrm{RAl}$ UT) is high but is not required for diagnosis unless patient is to be treated with RAI or subacute thyroiditis or Thyrotoxicosis factitia is suspected in which the RAI UT will be low.

There are three methods of treatment for Hyperthyroidism - Thyroidectomy, antithyroid drugs (PTU, Tapazole) and RAl. All three methods are reasonably good modalities but in general $\mathrm{RAl}$ treatment is preferred because of simplicity and low cost but at the risk of high incidence of Hypothyroidism which can occur with other treatments also. For young and pregnant patients, RAI treatment is avoided. Propranalol is usually added to therapeutic regimen to protect cardiovascular system.

\section{Few Comments about Thyroid Storm:}

The prompt clinical recognition of this condition is extremely important because lab data cannot be available instantaneously. Treatment has to be initiated right away as the disease carries a substantial morbidity and mortality. The clinical picture comprises of exacerbation of thyrotoxicity and high fever. If there is any doubt in the diagnosis, it is better 
to err on the side of overtreatment rather than undertreatment. Treatment consists of large doses of Propranalol, antithyroid drugs, steroids, oral or intravenous iodides, symptomatic management of fever, carefully looking for precipitating causes supportive care. In recent years $\mathrm{T}_{4}$ Toxicosis has been described in sick patients with high $\mathrm{T}_{4}$ and normal $\mathrm{T}_{3}$ R.1.A.

\section{Hypothyroidism}

Eriology

1. Primary thyroid failure - idiopathic atrophy, Hashimoto's thyroiditis, post Graves'disease with treatment particularly RAI treatment, subacute thyroiditis, congenital.

2. Secondary Hypothyroidism due to reduction of TSH in the Pituitary gland secondary to tumor, infarction, granuloma. Absence of high TSH with Hypothyroidism should lead one to suspect this condition. Normal and low levels of TSH cannot be differentiated because of methodology of the test. This accounts for less than $10 \%$ of all Hypothyroid patients.

3. Tertiary Hypothyroidism - This is a rare Hypothalamic disorder and can be diagnosed only by TRH-TSH test. In presence of Hypothyroidism, TSH, which is basically low, should respond to TRH administration to confirm this diagnosis.

\section{Diagnosis:}

The diagnosis of Hypothyroidism is relatively difficult clinically compared to Hyperthyroidism. Therefore, lab data plays an important role in its diagnosis. TSH is high in primary Hypothyroidism (Commonest form, more than 90\%) and is the first test to become abnormal. $\mathrm{T}_{7}$ is low and $\mathrm{T}_{3}$ R.I.A. plays a very limited role and is not required for the diagnosis. Patients with Hypothyroidism have mostly vague, ill defined non-specific complaints - malaise, weight gain, coarse dry skin, hoarseness of voice, sleepiness, puffiness of face and lower eye lids, alopecia, constipation, weakness, Brady-cardia, cold intolerance, etc. Very early cases of Hypothyroidism may have fewer or no symptoms and therefore lab gives very useful information. $\mathrm{T}_{7}$ and $\mathrm{TSH}$ confirm the diagnosis.

Treatment is simple and consists of slow replacement of thyroid hormones, commonly Lthyroxine, starting with 50 ug daily and building up the dose to 200 ug or so daily until $\mathrm{T}_{4}$ and TSH comes to normal. Patients with coronary artery disease need extra caution in very slow replacement and may feel better with mild Hypothyroidism.

Myxedema Coma is a serious disease of high mortality. A prompt clinical diagnosis expediting the treatment can cut down the mortality. Lab data cannot be available in such an emergency and therefore clinical impression is important although blood specimen should be saved for in vitro tests to make a retrospective diagnosis. Treatment consists of intravenous or Gastric tube administration of Lthyroxine, combating Hypothermia, steroids and identifying any other associated diseases.

At the time of diagnosis, if primary Hypothyroidism cannot be differentiated from Secondary, the use of steroids is important, lest the patient may go into Addisonian Crisis. The thyroid hormone will increase the metabolism of steroids resulting in its depletion.

\section{SUMMARY}

This was a very brief overview of Thyroid Physiology, interpretation of its function tests and management of thyroid diseases. In another issue the remaining topics - nodular thyroid disease, thyroid neoplasms and different types of thyroiditis will be presented.

\section{RECOMMENDED READING:}

I. Cavalieri R.R. Peripheral Metabolism of Thyroid Hormones. Thyroid Today. Vol. 3, No. 7 , Nov. 1980.

2. Surks M.I. Laboratory Aids in the Diagnosis of Hypothyroidism. Thyroid Today, Vol. 1, No. 4. Dec. 1977.

3. Chopra, I.J. Laboratory Aids in the Diagnosis of Hyperthyroidism. Vol. 1, No. 7, April, May, 1978.

4. The Thyroid (Harper \& Row, Publishers) Ed Werner, S.C. and Ingbar S.H., 1978.

5. Year Book in Endocrinology, 1981. 